\title{
Spectrum of Discrete Second-Order Difference Operator with Sign-Changing Weight and Its Applications
}

\author{
Ruyun Ma and Chenghua Gao \\ Department of Mathematics, Northwest Normal University, Lanzhou 730070, China \\ Correspondence should be addressed to Ruyun Ma; ruyun_ma@126.com
}

Received 13 March 2014; Accepted 28 March 2014; Published 13 April 2014

Academic Editor: Mustafa Kulenović

Copyright ( 2014 R. Ma and C. Gao. This is an open access article distributed under the Creative Commons Attribution License, which permits unrestricted use, distribution, and reproduction in any medium, provided the original work is properly cited.

Let $T>1$ be an integer, and let $\mathbb{T}=\{1,2, \ldots, T\}$. We discuss the spectrum of discrete linear second-order eigenvalue problems $\Delta^{2} u(t-1)+\lambda m(t) u(t)=0, t \in \mathbb{T}, u(0)=u(T+1)=0$, where $\lambda \neq 0$ is a parameter, $m: \mathbb{T} \rightarrow \mathbb{R}$ changes sign and $m(t) \neq 0$ on $\mathbb{T}$. At last, as an application of this spectrum result, we show the existence of sign-changing solutions of discrete nonlinear second-order problems by using bifurcate technique.

\section{Introduction}

Let $T>1$ be an integer, $\mathbb{T}=\{1,2, \ldots, T\}$. Let us consider the spectrum of the discrete second-order linear eigenvalue problem

$$
\begin{aligned}
\Delta^{2} u(t-1)+\lambda m(t) u(t) & =0, \quad t \in \mathbb{T}, \\
u(0)=u(T+1) & =0,
\end{aligned}
$$

where $\lambda \neq 0$ is a parameter, and $m$ changes sign on $\mathbb{T}$; that is, $m$ satisfies the following.

(H0) There exists a proper subset $\mathbb{T}_{+} \subset \mathbb{T}$, such that

$$
m(t)>0, \quad t \in \mathbb{T}_{+} ; \quad m(t)<0, \quad t \in \mathbb{T} \backslash \mathbb{T}_{+} .
$$

Let $n$ be the number of elements in $\mathbb{T}_{+}$. Then $n \in\{1, \ldots, T-1\}$.

In [1], Ince studied the second-order linear eigenvalue problem

$$
\begin{gathered}
u^{\prime \prime}(t)+\mu g(t) u(t)=0, \quad t \in(0,1), \\
u(0)=u(1)=0,
\end{gathered}
$$

where $g:[0,1] \rightarrow \mathbb{R}$ is continuous and changes sign. He obtained the following result.
Theorem A. Problem (4) has an infinite sequence of simple eigenvalues

$$
\begin{gathered}
-\infty \longleftarrow \cdots<\mu_{k,-}<\cdots<\mu_{2,-}<\mu_{1,-}<0<\mu_{1,+} \\
<\cdots<\mu_{k,+}<\cdots \longrightarrow+\infty
\end{gathered}
$$

and the eigenfunction corresponding to $\mu_{k, \pm}$ has exactly $k-1$ simple zeros in $(0,1)$.

This result has been extended to one-dimensional p-Laplacian operator by Anane et al. [2] and to the highdimensional case by Hess and Kato [3], Bongsoo and Brown [4], and Afrouzi and Brown [5]. Meanwhile, these spectrum results have been used to deal with several nonlinear problems; see, for example, [4-7] and the references therein.

For the discrete case, Atkinson [8] studied the discrete linear eigenvalue problems

$$
\begin{aligned}
& c(t) y(t+1) \\
& =(\lambda a(t)+b(t)) y(t)-c(t-1) y(t-1), \quad t \in \mathbb{T}, \\
& \quad y_{0}=0, \quad y(T+1)+l y(T)=0
\end{aligned}
$$

and obtained that (6) and (7) have exactly $T$ real eigenvalues, which can be ordered as $\lambda_{1}<\lambda_{2}<\cdots<\lambda_{T}$. Here $a(t)>0$, $c(t)>0$, and $l$ is some fixed real number. 
In 1995, Jirari [9] studied (6) with the more general boundary conditions

$$
y(0)+h y(1)=0, \quad y(T+1)+l y(T)=0,
$$

where $h, l \in \mathbb{R}$. He got that (6) and (8) have $T$ real eigenvalues, which can be ordered as $\lambda_{1}<\lambda_{2}<\cdots<\lambda_{T}$.

However, these two results do not give any information of the eigenfunctions of the linear eigenvalue problems (6) and (7) or (6) and (8).

In 1991, Kelley and Peterson [24] investigated the line eigenvalue problems

$$
\begin{gathered}
\Delta[p(t-1) \Delta y(t-1)]+q(t) y(t)+\lambda m(t) y(t)=0, \\
t \in \mathbb{T}, \\
y(0)=y(T+1)=0,
\end{gathered}
$$

where $p(t)>0$ on $\{0,1, \ldots, T\}, q(t)$ is defined and real valued on $\mathbb{T}$ and $m(t)>0$ on $\mathbb{T}$. They proved the following.

Theorem B. Problem (9) has exactly $T$ real and simple eigenvalues $\lambda_{k}, k \in \mathbb{T}$, which satisfies

$$
\lambda_{1}<\lambda_{2}<\cdots<\lambda_{T}
$$

and the eigenfunction corresponding to $\lambda_{k}$ has exactly $k-1$ simple generalized zeros.

Furthermore, when $m(t) \equiv 1$, Agarwal et al. [10] generalized the results of Theorem $B$ to the dynamic equations on time scales with Sturm-Liouville boundary condition. Moreover, under the assumption that the weight functions are not changing sign, several important results on linear Hamiltonian difference systems have also been established by Shi and Chen [11], Bohner [12], and the references therein.

However, there are few results on the spectrum of discrete second-order linear eigenvalue problems when $m(t)$ changes its sign on $\mathbb{T}$. In 2008, Shi and Yan [13] discussed the spectral theory of left definite difference operators when $m(t)$ may change its sign. However, they provided no information about the sign of the eigenvalues and no information about the corresponding eigenfunctions. Recently, Ma et al. [14] obtained that (1) and (2) have two principal eigenvalues $\lambda_{1,-}<$ $0<\lambda_{1,+}$ and they studied some corresponding discrete nonlinear problems.

It is the purpose of this paper to establish the discrete analogue of Theorem A for the discrete problems (1) and (2). More precisely, we will prove the following.

Theorem 1. Let $n$ be the number of elements in $\mathbb{T}_{+}$. Let (HO) hold and let $v \in\{+,-\}$. Then

(a) if $1 \leq n \leq T-1$, then (1) and (2) haveT real and simple eigenvalues, which can be ordered as follows:

$$
\begin{aligned}
\lambda_{T-n,-} & <\lambda_{T-n-1,-}<\cdots<\lambda_{1,-}<0<\lambda_{1,+} \\
& <\lambda_{2,+}<\cdots<\lambda_{n,+} ;
\end{aligned}
$$

(b) every eigenfunction $\psi_{k, v}$ corresponding to the eigenvalue $\lambda_{k, v}$ has exactly $k-1$ simple generalized zeros.
The rest of the paper is arranged as follows. In Section 2, some preliminaries will be given including Lagrange-type identities. In Section 3, we develop a new method to count the number of negative and positive eigenvalues of (1) and (2), which enable us to prove Theorem 1. Finally in Section 4, we apply our spectrum theory and the Rabinowitz's bifurcation theorem to consider the existence of sign-changing solutions of discrete nonlinear problems

$$
\begin{gathered}
\Delta^{2} u(t-1)+r m(t) f(u(t))=0, \quad t \in \mathbb{T}, \\
u(0)=u(T+1)=0,
\end{gathered}
$$

where $r \neq 0$ is a real parameter, $m: \mathbb{T} \rightarrow \mathbb{R}$ changes its sign, $m(t) \neq 0$ on $\mathbb{T}$, and $f: \mathbb{R} \rightarrow \mathbb{R}$ is continuous.

Remark 2. There is also much literature dealing with difference equations similar to (12) subject to various boundary value conditions. We refer to [15-22] and the references therein. However, the weight $m(t)>0$ in these papers.

\section{Preliminaries}

Recall that $\mathbb{T}=\{1, \ldots, T\}$. Let $\widehat{\mathbb{T}}=\{0,1, \ldots, T, T+1\}, X=$ $\{u: \widehat{\mathbb{T}} \rightarrow \mathbb{R} \mid u(0)=u(T+1)=0\}$. Then $X$ is a Banach space under the norm $\|u\|_{X}=\max _{t \in \hat{\mathbb{T}}}|u(t)|$. Let $Y=\{u \mid u$ : $\mathbb{T} \rightarrow \mathbb{R}\}$. Then $Y$ is a Banach space under the norm $\|u\|_{Y}=$ $\max _{t \in \mathbb{T}}|u(t)|$.

Definition 3 (see [22]). Suppose that a function $y: \widehat{\mathbb{T}} \rightarrow \mathbb{R}$. If $y(t)=0$, then $t$ is a zero of $y$. If $y(t)=0$ and $y(t-1) y(t+1)<0$ for some $t \in\{2, \ldots, T-1\}$, then $t$ is a simple zero of $y$. If $y(t) y(t+1)<0$ for some $t \in\{1, \ldots, T-1\}$, then $y$ has a node at the point

$$
s=\frac{t y(t+1)-(t+1) y(t)}{y(t+1)-y(t)} \in(t, t+1) .
$$

The nodes and simple zeros of $y$ are called the simple generalized zeros of $y$.

To find the eigenvalue of (1) and (2), we rewrite (1) as follows:

$$
u(t+1)=(2-\lambda m(t)) u(t)-u(t-1), \quad t \in \mathbb{T} .
$$

From (15), it can be seen that if $\lambda$ is an eigenvalue of (1) and (2), then $u(1) \neq 0$. Without loss of generality, we suppose that

$$
u(1)=1 \text {. }
$$

Further, from (15), (2), and (16), for each $t \in \mathbb{T}, u(t)$ is precisely a polynomial of degree $t-1$ of $\lambda$, we denote it by $u(t, \lambda)$.

Lemma 4 (Lagrange-type identities). For $t \in \mathbb{T}$,

$$
\begin{aligned}
(\lambda-\mu) & \sum_{s=1}^{t} m(s) u(s, \lambda) u(s, \mu) \\
= & \left|\begin{array}{cc}
u(t+1, \mu) & u(t+1, \lambda) \\
u(t, \mu) & u(t, \lambda)
\end{array}\right| .
\end{aligned}
$$


Proof. We write (15) for the two arguments in full, giving

$$
\begin{aligned}
& u(t+1, \lambda)=(2-\lambda m(t)) u(t, \lambda)-u(t-1, \lambda), \\
& u(t+1, \mu)=(2-\mu m(t)) u(t, \mu)-u(t-1, \mu) .
\end{aligned}
$$

Multiplying, respectively, by $u(t, \mu), u(t, \lambda)$ and subtracting, we have

$$
\begin{aligned}
\left|\begin{array}{cc}
u(t+1, \lambda) & u(t+1, \mu) \\
u(t, \lambda) & u(t, \mu)
\end{array}\right|= & (\mu-\lambda) m(t) u(t, \lambda) u(t, \mu) \\
& +\left|\begin{array}{cc}
u(t, \lambda) & u(t, \mu) \\
u(t-1, \lambda) & u(t-1, \mu)
\end{array}\right|
\end{aligned}
$$

and putting $t=1$ and recalling that $u(0, \lambda)=u(0, \mu)=0$, we derive (17) with $t=1$. Induction over $t$ then yields (17) from (19) in the general case.

Corollary 5. For $t \in \mathbb{T}$,

$$
\sum_{s=1}^{t} m(s)(u(s, \lambda))^{2}=\left|\begin{array}{cc}
u(t+1, \lambda) & u^{\prime}(t+1, \lambda) \\
u(t, \lambda) & u^{\prime}(t, \lambda)
\end{array}\right| .
$$

Proof. Dividing (17) by $\mu-\lambda$ and making $\mu \rightarrow \lambda$ for fixed $\lambda$, then we get the desired result.

Corollary 6. For $t \in \mathbb{T}$, and complex $\lambda$,

$$
\begin{aligned}
& \sum_{s=1}^{t} m(s)|u(s, \lambda)|^{2} \\
& =(2 i \operatorname{Im} \lambda)^{-1}\left|\begin{array}{cc}
u(t+1, \bar{\lambda}) & u(t+1, \lambda) \\
u(t, \bar{\lambda}) & u(t, \lambda)
\end{array}\right| .
\end{aligned}
$$

Proof. Set $\mu=\bar{\lambda}$ in (17). Then (21) is obtained.

\section{Spectrum of (1) and (2)}

Lemma 7. For $t \in \mathbb{T}$, if $(\lambda, u)$ is a solution of

$$
\Delta^{2} u(s-1)+\lambda m(s) u(s)=0, \quad s \in\{1,2, \ldots, t\},
$$

satisfying $u(0)=0, u(t) u(t+1) \leq 0$, and $u \neq \equiv$ on $\mathbb{T}$, then

$$
\sum_{s=1}^{t} m(s)(u(s))^{2} \neq 0
$$

Moreover, if $\lambda>0$, then $\sum_{s=1}^{t} m(s)(u(s))^{2}>0$. If $\lambda<0$, then $\sum_{s=1}^{t} m(s)(u(s))^{2}<0$.
Proof. It is easy to see that $\lambda \neq 0$. Now, multiplying (22) by $u(s)$, then we get that

$$
\begin{aligned}
\lambda \sum_{s=1}^{t} m(s)(u(s))^{2} & \\
\quad= & \sum_{s=1}^{t}-\Delta^{2} u(s-1) u(s) \\
= & \sum_{s=1}^{t} \Delta u(s-1) u(s)-\sum_{s=1}^{t} \Delta u(s) u(s) \\
= & \sum_{s=0}^{t-1} \Delta u(s) u(s+1)-\sum_{s=1}^{t} \Delta u(s) u(s) \\
= & \sum_{s=1}^{t-1} \Delta u(s) u(s+1) \\
& -\sum_{s=1}^{t-1} \Delta u(s) u(s)+u(1) \Delta u(0)-u(t) \Delta u(t) \\
= & \sum_{s=1}^{t-1}(\Delta u(s))^{2}+(u(1))^{2}+(u(t))^{2}-u(t) u(t+1),
\end{aligned}
$$

which implies the desired result.

Now, we prove some oscillatory properties.

Lemma 8. For $t \in\{1,2, \ldots, T+1\}$, the polynomial $u(t, \lambda)$ has precisely $t-1$ real and simple zeros.

Proof. This proof is divided into two steps.

Step 1 (each zero of $u(t, \lambda)$ is real). Suppose on the contrary that $u(t, \lambda)$ has a complex zero $\lambda_{0}$; then $u\left(t, \lambda_{0}\right)=u\left(t, \bar{\lambda}_{0}\right)=0$. Furthermore,

$$
\left|\begin{array}{cc}
u\left(t, \bar{\lambda}_{0}\right) & u\left(t, \lambda_{0}\right) \\
u\left(t-1, \bar{\lambda}_{0}\right) & u\left(t-1, \lambda_{0}\right)
\end{array}\right|=0
$$

On the other hand, if $\lambda_{0}$ is a zero of $u(t, \lambda)$, then $\lambda_{0}$ is an eigenvalue of the linear eigenvalue problem

$$
\begin{gathered}
\Delta^{2} u(s-1)+\lambda m(s) u(s)=0, \quad s \in\{1,2, \ldots, t-1\}, \\
u(0)=u(t)=0 .
\end{gathered}
$$

Now, by Lemma 7 and Corollary 6, we get that the above determinant does not equal zero, which is a contradiction. Hence, the zeros of $u(t, \lambda)$ are all real for $t \in \mathbb{T}$.

Step 2 (all of the zeros of $u(t, \lambda)$ are simple). Suppose on the contrary that $u(t, \lambda)$ has a multiple zeros $\lambda_{*}$, necessarily real. Then $u\left(t, \lambda_{*}\right)=0$ and $u^{\prime}\left(t, \lambda_{*}\right)=0$. Moreover,

$$
\left|\begin{array}{cc}
u^{\prime}\left(t, \lambda_{*}\right) & u\left(t, \lambda_{*}\right) \\
u^{\prime}\left(t-1, \lambda_{*}\right) & u\left(t-1, \lambda_{*}\right)
\end{array}\right|=0 .
$$

However, by Lemma 7 and Corollary 5, we get that the above determinant does not equal zero, a contradiction. 
Lemma 9 (see [14]). Let $n \in\{1, \ldots, T-1\}$. Then (1) and (2) have two principal eigenvalues $\lambda_{1,+}>0>\lambda_{1,-}$ and the corresponding eigenfunctions do not change their sign.

From Lemma 8, it follows that the spectra of (1) and (2) consist of $T$ real eigenvalues. Furthermore, by Lemma 9, there exists $p \in\{1, \ldots, T-1\}$ such that such $T$ real eigenvalues can be ordered as follows:

$$
\lambda_{T-p,-}<\lambda_{T-p-1,-}<\cdots<\lambda_{1,-}<0<\lambda_{1,+}<\cdots<\lambda_{p,+}
$$

Define $L: X \rightarrow Y$ by

$$
L u(t)=-\Delta^{2} u(t-1), \quad u \in X .
$$

Then we get the following.

Lemma 10. Let $v \in\{+,-\}$. Then $\operatorname{dim} \operatorname{ker}\left(L-\lambda_{k, v} m I\right)=1$, where I denotes the identity operator.

Proof. Suppose that $\varphi_{k, v} \in X$ and $\phi_{k, v} \in X$ are two eigenfunctions corresponding to $\lambda_{k, v}$. Then $\varphi_{k, v}(0)=\phi_{k, v}(0)=0$ and there exists a constant $c \in \mathbb{R}$ such that $\varphi_{k, v}(1)=c \phi_{k, v}(1) \neq 0$. Now, by the recurrence relation (15), we get that $\varphi_{k, v}(t)=$ $c \phi_{k, v}(t)$ for $t \in \widehat{\mathbb{T}}$.

By Lemmas 8-10, all of the eigenvalues of (1) and (2) are real and simple. Now, for fixed $\lambda$, let us investigate the number of sign-changing times of the following sequence,

$$
u(1, \lambda), u(2, \lambda), \ldots, u(T, \lambda) .
$$

So far, $u(t, \lambda)$ has only been defined for integral values of $t, t=0,1, \ldots, T+1$. We extend it to a continuous function $u(x, \lambda), 0 \leq x \leq T+1$, specifically, for $t \leq x \leq t+1$, $u(x, \lambda)=(u(t+1, \lambda)-u(t, \lambda))(x-t)+u(t, \lambda)$ to be a linear function of $x$.

Lemma 11. For fixed real $\lambda$, the zeros of $u(x, \lambda), 0 \leq x \leq T+1$ are simple.

Proof. Suppose that $x^{\prime}$ is a zero of $u(x, \lambda)$. Now, the proof can be divided into two cases.

Case 1. If $x_{0} \in \mathbb{T}$, then by virtue of (15), we get that $u\left(x_{0}+\right.$ $1, \lambda) u\left(x_{0}-1, \lambda\right)<0$.

Case 2. If $x_{0} \in(t, t+1)$ for some $t \in \mathbb{T}$. Then $(\partial / \partial x) u(x, \lambda)$ exists at $x=x_{0}$ and is not zero by the definition of $u(x, \lambda)$.

Using the same method used in [1, Page 102], we may prove the following.

Lemma 12. Let $x(\lambda)$ be the zeros of $u(t+1, \lambda)$. Then $x(\lambda)$ is a continuous function of $\lambda$.

Lemma 13. For $\lambda>0$, the zeros, $x(\lambda)$, of $u(x, \lambda), x \in(0, T+1]$ is a decreasing function of $\lambda$. For $\lambda<0, x(\lambda)$ is an increasing function of $\lambda$ for $x \in(0, T+1]$.
Proof. Let the zero $x(\lambda)$ occur in $(t, t+1]$. Since $u(x, \lambda)$ is linear in $(t, t+1]$, the location of this zero is given by

$$
x(\lambda)=t+\frac{u(t, \lambda)}{u(t, \lambda)-u(t+1, \lambda)} ;
$$

conversely, $x(\lambda)$ as given by this equation will actually be a zero of $u(x, \lambda)$ if $u(t, \lambda) \neq u(t+1, \lambda)$ and if $x(\lambda)$ so the given falls in the interval $(t, t+1]$. If we differentiate the right of (31), with respect to $\lambda$, the result is found to be

$$
x^{\prime}(\lambda)=\frac{(\partial / \partial \lambda) u(t+1, \lambda) u(t, \lambda)-u(t+1)(\partial / \partial \lambda) u(t, \lambda)}{(u(t, \lambda)-u(t+1, \lambda))^{2}},
$$

and this does not equal zero by Lemma 7 and Corollary 5. Furthermore, for $\lambda>0, x^{\prime}(\lambda)<0$ and for $\lambda<0, x^{\prime}(\lambda)>0$. This combines with the continuity of $x(\lambda)$, and we get the desired result.

Now, we can set up the oscillatory characterization of the eigenvalues of (1) and (2).

Lemma 14. Let $v \in\{+,-\}$. The sequence

$$
\{u(0, \lambda), u(1, \lambda), u(2, \lambda), \ldots, u(T+1, \lambda)\}
$$

exhibits for $\lambda_{1,-} \leq \lambda \leq \lambda_{1,+}$ no changes of sign; for $\left|\lambda_{k, v}\right|<\lambda \leq$ $\left|\lambda_{k+1, v}\right|$, exactly $k$ changes sign; for $\lambda>\lambda_{p,+}$, exactly $p$ changes sign; for $\lambda<\lambda_{T-p,-}$, exactly $T-p$ changes sign.

Proof. To prove the times of changes of sign of (33), it is equivalent to find the number of zeros of $u(x, \lambda), x \in(1, T+$ $1)$. We only deal with the case that $v=+$; the case $v=-$ is similar.

By Lemma 12, for $k=1,2,3, \ldots, p-1$, there exist $p-1$ functions $x_{k,+}(\lambda)$, which satisfy $x_{k,+}\left(\lambda_{k,+}\right)=T+1$ and are all decreasing function of $\lambda$; moreover, for fixed $\lambda$, there are the zeros of $u(x, \lambda)$. Since $u(0, \lambda)=0$ and $u(1, \lambda)=1$, it follows that $x_{k,+}(\lambda) \in(1, T+1)$ for $\lambda>\lambda_{k,+}$.

For $0 \leq \lambda \leq \lambda_{1,+}$, by Lemma 8 , we get that $u(x, \lambda)$ does not have a zero in $(1, T+1)$.

Let $\lambda_{\diamond} \in\left(\lambda_{1,+}, \lambda_{2,+}\right]$ be arbitrary. Since $x_{1,+}(\lambda)$ is continuous and decreasing, $x_{1,+}(\lambda)$ will intersect with $\lambda=\lambda_{\diamond}$ at $\left(\lambda_{\diamond}, x_{1,+}\left(\lambda_{\diamond}\right)\right)$. Moreover, $x_{1,+}\left(\lambda_{1,+}\right)=T+1$ and $\lambda_{\diamond}>\lambda_{1,+}$, which implies that $x_{1,+}\left(\lambda_{\diamond}\right)<T+1$. Thus, for $\lambda=\lambda_{2,+},(33)$ changes its sign exactly one time.

Now, we claim that for the same $\lambda, x_{k,+}(\lambda)$ and $x_{k+1,+}(\lambda)$ have no common zero for each $k \in\{1,2, \ldots, p-1\}$.

Suppose the contrary, then there exists $\lambda^{*} \in\left(\lambda_{2,+}, \lambda_{p,+}\right)$ such that

$$
\lambda^{*}=\min \left\{\lambda \mid x_{k,+}(\lambda)=x_{k+1,+}(\lambda), k=1,2, \ldots, p-2\right\} .
$$

Let

$$
k^{*}=\min \left\{k \mid x_{k,+}\left(\lambda^{*}\right)=x_{k+1,+}\left(\lambda^{*}\right), k=1,2, \ldots, p-2\right\} .
$$

Then, for $\lambda \leq \lambda^{*}, x_{k^{*},+}(\lambda)$ is the $k^{*}$ th zero of $u(x, \lambda)$. 
Case 1. If there exists $t_{0} \in \mathbb{T}$ such that $x_{k^{*}+1,+}\left(\lambda^{*}\right)=$ $x_{k^{*},+}\left(\lambda^{*}\right) \in\left(t_{0}, t_{0}+1\right)$, then by the definition of $u(x, \lambda)$, we obtain that the signs of $u\left(t_{0}, \lambda^{*}\right)$ and $u\left(t_{0}+1, \lambda^{*}\right)$ are opposite. Without loss of generality, suppose that $u\left(t_{0}, \lambda^{*}\right)>0$ and $u\left(t_{0}+1, \lambda^{*}\right)<0$.

Now, consider the variation of $x_{k^{*}+1,+}(\lambda)$ when $\lambda$ varies. Take $\varepsilon>0$ sufficiently small, by the continuity of $x_{k^{*}+1,+}(\lambda)$ with respect to $\lambda$, for $\lambda \in\left(\lambda^{*}-\varepsilon, \lambda^{*}\right), x_{k^{*}+1,+}(\lambda) \in\left(t_{0}, t_{0}+1\right)$, and also $u\left(t_{0}, \lambda\right)>0, u\left(t_{0}+1, \lambda\right)<0$ since $u(t, \lambda)$ is a continuous function of $\lambda$. However, for $\lambda \in\left(\lambda^{*}-\varepsilon, \lambda^{*}\right)$, $x_{k^{*}+1,+}(\lambda)$ is the $\left(k^{*}+1\right)$ th zero of $u(x, \lambda)$, which implies that $u\left(t_{0}, \lambda\right)<0, u\left(t_{0}+1, \lambda\right)>0$, a contradiction.

Case 2. If there exists $t_{0} \in \mathbb{\mathbb { T }}$ such that $x_{k^{*}+1,+}\left(\lambda^{*}\right)=$ $x_{k^{*},+}\left(\lambda^{*}\right)=t_{0}$, then we consider the sign of $u\left(t_{0}-1, \lambda^{*}\right)$ and $u\left(t_{0}+1, \lambda^{*}\right)$, and we can get the similar contradiction as in Case 1.

This claim implies that, for the same $\lambda, x_{k,+}(\lambda), k=$ $1,2, \ldots, p-1$ do not intersect with each other. Thus, for $\lambda_{k-1,+}<\lambda_{\diamond} \leq \lambda_{k,+}$, there are $k-1$ functions: $x_{j,+}, j=$ $1,2, \ldots, k-1$ which intersect with $\lambda=\lambda_{\diamond}$ in $(1, T+1)$ at $k-1$ different points; that is, for $\lambda_{k-1,+}<\lambda_{\diamond} \leq \lambda_{k,+}, u\left(x, \lambda_{\diamond}\right)$ has exactly $k-1$ zeros.

This completes the proof.

Lemma 15. Problems (1) and (2) have exactly $n$ positive eigenvalues and exactly $T-n$ negative eigenvalues.

Proof. First we show that $\psi_{p,+}$ changes its sign exactly $n-1$ times.

Let us consider the following $T+2$ ordered polynomials:

$$
\begin{aligned}
& u(0, \lambda)=0, \quad u(1, \lambda)=1, \\
& u(2, \lambda)=(-1)^{1} m(1) \lambda+Q_{1}(\lambda), \\
& u(3, \lambda)=(-1)^{2} m(1) m(2) \lambda^{2}+Q_{2}(\lambda), \\
& \vdots \\
& u(T+1, \lambda)=(-1)^{T} m(1) \cdots m(T) \lambda^{T}+Q_{T}(\lambda) \\
& =(-1)^{n}|m(1)| \cdots|m(T)| \lambda^{T}+Q_{T}(\lambda),
\end{aligned}
$$

where $Q_{j}(\lambda)$ is a polynomial of degree precisely $j-1$ of $\lambda$.

Observation 1. Consider

$$
\operatorname{NSC}(\lambda)=\operatorname{NSC}\left(\lambda_{p,+}\right), \quad \forall \lambda>\lambda_{p,+},
$$

where $\operatorname{NSC}(\lambda)$ is the number of sign changes of

$$
\{u(0, \lambda), u(1, \lambda), \ldots, u(T, \lambda), u(T+1, \lambda)\} .
$$

Observation 2. For $j \in\{2, \ldots, T\}$, denoted by $\Gamma(j)$, the number of the elements in the set

$$
\left\{m_{k} \mid m_{k}>0 \text { for some } k \in\{1, \ldots, j\}\right\} .
$$

Then $\Gamma(T)=n$. Now

$$
\begin{aligned}
\operatorname{sgn} & (u(j+1, \lambda)) \\
= & \operatorname{sgn}\left((-1)^{j} m_{1} \cdots m_{j} \lambda^{j}\right) \\
= & (-1)^{j+(j-\Gamma(j))}=(-1)^{\Gamma(j)}, \quad j \in\{1, \ldots, T\},
\end{aligned}
$$

if $\lambda>0$ is large enough. Since

$$
\left\{0,1,(-1)^{\Gamma(2)}, \ldots,(-1)^{\Gamma(T)},(-1)^{\Gamma(T+1)}\right\}
$$

changes sign exactly $\Gamma(T)$ times, it follows that

$$
\{u(0, \lambda), u(1, \lambda), \ldots, u(T, \lambda), u(T+1, \lambda)\}
$$

changes sign exactly $\Gamma(T)$ times as $\lambda>0$ is large enough; that is,

$$
\operatorname{NSC}(\lambda)=\Gamma(T) .
$$

This together with (37) implies that $\operatorname{NSC}\left(\lambda_{p,+}\right)=n$. So, $\psi_{p,+}$ changes its sign exactly $n$ times.

Next, using the result of first step and Lemma 14, it follows that $p=n$.

Proof of Theorem 1. From Lemma 7-Lemma 15, the results of Theorem 1 hold.

\section{Application}

As an application, we consider the existence of sign-changing solutions of the discrete nonlinear boundary value problems (12), (13).

In this section we suppose that

(H1) $f \in C([0, \infty),(-\infty,+\infty))$ with $f(0)=f\left(s_{1}\right)=$ $f\left(s_{2}\right)=0,0<s_{1} \leq s_{2} ; f(s)>0$ for $s \in\left(0, s_{1}\right) \cup$ $\left(s_{2},+\infty\right), f(s)<0$ for $s \in\left(s_{1}, s_{2}\right)$;

(H2) there exist $f_{0}, f_{\infty} \in(0, \infty)$ such that

$$
f_{0}=\lim _{|s| \rightarrow 0^{+}} \frac{f(s)}{s}, \quad f_{\infty}=\lim _{|s| \rightarrow \infty} \frac{f(s)}{s} ;
$$

for simplicity, we give some notations at first.

For $k \geq 1, v \in\{+,-\}$, let $S_{k}^{v}$ denote the set of functions in $X$ such that

(1) $u$ has exactly $k-1$ simple generalized zeros in $(1, T+1)$;

(2) $v u(1)>0$.

Define $S_{k}=S_{k}^{+} \cup S_{k}^{-}$. They are disjoint in $X$. Finally, let $\Psi_{k}^{ \pm}=\mathbb{R} \times S_{k}^{ \pm}$and let $\Psi_{k}=\mathbb{R} \times S_{k}$.

Theorem 16. Suppose that (H0), (H1), and (H2) hold. Assume that $f_{0}<f_{\infty}$. Then

(i) if

$$
r \in\left(\frac{\lambda_{k,+}}{f_{0}},+\infty\right), \quad k \in\{1,2, \ldots, n\},
$$


problems (12) and (13) have at least four sign-changing solutions $u_{k, 1} \in S_{k}^{+}, u_{k, 2} \in S_{k}^{+}, u_{k, 3} \in S_{k}^{-}$, and $u_{k, 4} \in S_{k}^{-}$;

(ii) if

$$
r \in\left(-\infty, \frac{\lambda_{l,-}}{f_{0}}\right) \quad l \in\{1,2, \ldots, T-n\},
$$

problems (12) and (13) have at least four sign-changing solutions $u_{1,1} \in S_{l}^{+}, u_{l, 2} \in S_{l}^{+}, u_{l, 3} \in S_{l}^{-}$, and $u_{l, 4} \in S_{l}^{-}$.

Moreover, for $r \in\left(\lambda_{k,+} / f_{\infty}, \lambda_{k,+} / f_{0}\right]$, there also exist at least two sign-changing solutions $u_{k, 1} \in S_{k}^{+}$and $u_{k, 2} \in S_{k}^{-}$; meanwhile, for $r \in\left[\lambda_{l,-} / f_{0}, \lambda_{l,-} / f_{\infty}\right)$, there also exist at least two sign-changing solutions $u_{l, 1} \in S_{l}^{+}$and $u_{l, 2} \in S_{l}^{-}$.

Theorem 17. Suppose that (H0), (H1), and (H2) hold. Assume that $f_{\infty}<f_{0}$. Then

(i) if

$$
r \in\left(\frac{\lambda_{k,+}}{f_{\infty}},+\infty\right), \quad k \in\{1,2, \ldots, n\},
$$

problems (12) and (13) have at least four sign-changing solutions $u_{k, 1} \in S_{k}^{+}, u_{k, 2} \in S_{k}^{+}, u_{k, 3} \in S_{k}^{-}$, and $u_{k, 4} \in S_{k}^{-}$;

(ii) if

$$
r \in\left(-\infty, \frac{\lambda_{l,-}}{f_{\infty}}\right) \quad l \in\{1,2, \ldots, T-n\},
$$

problems (12) and (13) have at least four sign-changing solutions $u_{1,1} \in S_{l}^{+}, u_{l, 2} \in S_{l}^{+}, u_{l, 3} \in S_{l}^{-}$, and $u_{l, 4} \in S_{l}^{-}$.

Moreover, for $r \in\left(\lambda_{k,+} / f_{0}, \lambda_{k,+} / f_{\infty}\right]$, there exist at least two sign-changing solutions $u_{k, 1} \in S_{k}^{+}$and $u_{k, 2} \in S_{k}^{-}$; meanwhile, for $r \in\left[\lambda_{l,-} / f_{\infty}, \lambda_{l,-} / f_{0}\right)$, there also exist at least two sign-changing solutions $u_{l, 1} \in S_{l}^{+}$and $u_{l, 2} \in S_{l}^{-}$.

If condition (H2) is replaced by

$(\overline{\mathrm{H}} 2) f_{0} \in(0, \infty), f_{\infty}=\infty$,

then we obtain the following result.

Theorem 18. Let (HO), (H1), and $(\overline{\mathrm{H}} 2)$ hold. Then (12) and (13) have a sign-changing solution in $S_{k}^{v},(k=1,2, \ldots, n)$ if and only if $r \neq 0$. Moreover, for $r \in\left(-\infty, \lambda_{l,-} / f_{0}\right)$, there exist at least two solutions $u_{l, 1} \in S_{l}^{+}$and $u_{l, 2} \in S_{l}^{-}$, and there also exist at least two solutions $u_{k, 1} \in S_{k}^{+}$and $u_{k, 2} \in S_{k}^{-}$for $r \in$ $\left(\lambda_{k,+} / f_{0},+\infty\right)$.

Recall that $L: X \rightarrow Y$; then

$$
L u(t)=-\Delta^{2} u(t-1) .
$$

Let $\zeta \in C(\mathbb{R}, \mathbb{R})$ be such that

$$
f(u)=f_{0} u+\zeta(u) .
$$

Clearly

$$
\lim _{|u| \rightarrow 0} \frac{\zeta(u)}{u}=0
$$

Let us consider

$$
L u-\lambda m(t) r f_{0} u-\lambda m(t) r \zeta(u)=0
$$

as a bifurcation problem from the trivial solution $u \equiv 0$.

Equation (52) can be converted to the equivalent equation

$$
u(t)=\lambda L^{-1}\left[m(\cdot) r f_{0} u(\cdot)+m(\cdot) r \zeta(u(\cdot))\right](t) .
$$

Further we note that $\left\|L^{-1}[m(\cdot) \zeta(u(\cdot))]\right\|=o(\|u\|)$ for $u$ near 0 in $X$, since

$$
\begin{aligned}
\left\|L^{-1}[m(\cdot) \zeta(u(\cdot))]\right\| & =\max _{t \in \mathbb{\mathbb { T }}}\left|\sum_{s=1}^{T} G(t, s) m(s) \zeta(u(s))\right| \\
& \leq C \cdot \max _{s \in \mathbb{T}}|m(s)|\|\zeta(u(\cdot))\|,
\end{aligned}
$$

where $C=\max _{t \in \hat{\mathbb{\pi}}} \sum_{s=1}^{T} G(t, s)$ and

$$
G(t, s)=\frac{1}{T+1} \begin{cases}(T+1-t) s, & 0 \leq s \leq t \leq T+1 \\ (T+1-s) t, & 0 \leq t \leq s \leq T+1\end{cases}
$$

Lemma 19. Suppose that $(r, u)$ is a nontrivial solution of (12) and (13); then there exists $k_{0} \in\{1,2, \ldots, \max \{n, T-n\}\}$ such that

$$
u \in S_{k_{0}}^{v}
$$

Proof. Suppose on the contrary that for every $k \in\{1,2, \ldots$, $\max \{n, T-n\}\}$

$$
u \notin S_{k}^{v}
$$

Then there exists $t_{0} \in \mathbb{T}$ such that

$$
u\left(t_{0}\right)=0, \quad u\left(t_{0}-1\right) u\left(t_{0}+1\right) \geq 0 .
$$

Since $u \neq \equiv$ on $\widehat{\mathbb{T}}$, we may assume that

$$
\left|u\left(t_{0}-1\right)\right|+\left|u\left(t_{0}+1\right)\right|>0 .
$$

On the other hand, it follows from (52) and $f(0)=0$ that

$$
-\Delta^{2} u\left(t_{0}-1\right)=\lambda\left[r m\left(t_{0}\right) f\left(u\left(t_{0}\right)\right)\right]=0,
$$

which implies that

$$
u\left(t_{0}+1\right)-2 u\left(t_{0}\right)+u\left(t_{0}-1\right)=0 .
$$

However, by (59) and the fact $u\left(t_{0}\right)=0$, we get

$$
u\left(t_{0}+1\right) u\left(t_{0}-1\right)<0,
$$

which contradicts (58).

Now, the results of Rabinowitz [23] for (52) can be stated as follows. 
(i) For each integer $k \in\{1,2, \ldots, n\}$, there exists a continuum $\mathscr{C}_{k,+}^{v}$ of solutions to (52) joining $\left(\lambda_{k,+} / r f_{0}, 0\right)$ to infinity in $\Psi_{k}^{v}$. Moreover, $\mathscr{C}_{k,+}^{v} \backslash\left\{\left(\lambda_{k,+} / r f_{0}, 0\right)\right\} \subset \Psi_{k}^{v}$.

(ii) For each integer $l \in\{1,2, \ldots, T-n\}$, there exists a continuum $\mathscr{C}_{l,-}^{v}$ of solutions to (52) joining $\left(-\lambda_{l,-} / r f_{0}, 0\right)$ to infinity in $\Psi_{l}^{\nu}$. Moreover, $\mathscr{C}_{l,-}^{\nu} \backslash\left\{\left(-\lambda_{l,-} / r f_{0}, 0\right)\right\} \subset \Psi_{l}^{\nu}$.

Lemma 20. Suppose that (HO), (H1), and (H2) hold. Then for $(r, u) \in \mathscr{C}_{k,+}^{v} \cup \mathscr{C}_{l,-}^{v}$

$$
0<u(t)<s_{1}, \quad t \in \mathbb{T} .
$$

Proof. Suppose on the contrary that there exists $(r, u) \in \mathscr{C}_{k,+}^{v}$ such that

$$
\max _{t \in \mathbb{T}} u(t)=s_{1} .
$$

By (H0), (H1), and (H2), there exists $b \geq 0$ such that $m(t) f(s)+b s$ is strictly increasing in $s$ for $s \in\left[0, s_{1}\right]$. Then

$$
-\Delta^{2} u(t-1)+r b u(t)=r(m(t) f(u(t))+b u(t)), \quad t \in \mathbb{T},
$$

and since $\Delta^{2} s_{1}=0=f\left(s_{1}\right)$,

$$
-\Delta^{2} s_{1}(t-1)+r b s_{1}=r\left(m(t) f\left(s_{1}\right)+b s_{1}\right), \quad t \in \mathbb{T} .
$$

Subtracting, we get

$$
-\Delta^{2}\left(s_{1}-u\right)(t-1)+r b\left(s_{1}-u\right)>0, \quad t \in \mathbb{T} .
$$

Let $w=s_{1}-u$, and applying boundary value problem (12) and (13), we have

$$
\begin{gathered}
-\Delta w(t-1)+r b w(t)>0, \quad t \in \mathbb{T}, \\
w(0)=w(T+1)=0 .
\end{gathered}
$$

Let $e: \mathbb{T} \rightarrow(0, \infty)$, such that

$$
-\Delta^{2} w(t-1)+r b w(t)=e(t)>0, \quad t \in \mathbb{T} .
$$

Let $G_{1}(t, s)$ be the Green function of the boundary value problem

$$
\begin{gathered}
-\Delta^{2} w(t-1)+r b w(t)=e(t)>0, \quad t \in \mathbb{T}, \\
w(0)=w(T+1)=0 .
\end{gathered}
$$

From $r>0, b>0$, applying Theorem 6.8 and Corollary 6.7 of [24], we have $G_{1}(t, s)>0, \forall t, s \in \mathbb{T}$ and $G(0, s)=G(T+$ $1, s)=0$ for $s \in \mathbb{T}$.

Problem (70) is equivalent to

$$
w(t)=\sum_{s=1}^{T} G_{1}(t, s) e(s) d s, \quad t \in \widehat{\mathbb{T}} .
$$

By using the positivity of $G_{1}(t, s)$ and $e(s)$, we have

$$
w(t)>0, \quad t \in \mathbb{T} ;
$$

that is, $s_{1}>u(t), t \in \mathbb{T}$. This contradicts (64).
There exists $(r, u) \in \mathscr{C}_{l,-}^{v}$ such that

$$
\max _{t \in \mathbb{I}} u(t)=s_{1} .
$$

Note that in this case $r<0$, so we can choose $\widetilde{b} \geq 0$ such that $m(t) f(s)-\widetilde{b} s$ is strictly decreasing in $s$ for $s \in\left[0, s_{1}\right]$. Then

$$
-\Delta^{2} u(t-1)-r \widetilde{b} u=r(m(t) f(u)-\widetilde{b} u), \quad t \in \mathbb{T},
$$

and since $\Delta^{2} s_{1}(t-1)=0=f\left(s_{1}\right)$,

$$
-\Delta^{2} s_{1}(t-1)-r \widetilde{b} s_{1}>r\left(m(t) f\left(s_{1}\right)-\widetilde{b} s_{1}\right), \quad t \in \mathbb{T} .
$$

Subtracting, we get

$$
-\Delta^{2}\left(s_{1}-u\right)(t-1)-r \widetilde{b}\left(s_{1}-u\right)(t)>0, \quad t \in \mathbb{T} .
$$

Let $w=s_{1}-u$, and applying boundary value condition (13), we have

$$
\begin{gathered}
-\Delta^{2} w(t-1)-r \tilde{b} w(t)>0, \quad t \in \mathbb{T}, \\
w(0)=w(T+1)=0 .
\end{gathered}
$$

Similar to the above proof, we have $s_{1}>u(t), t \in \mathbb{T}$. This contradicts (73).

In the following we will investigate other sign-changing solutions of problems (1) and (2).

Let $\xi \in C(\mathbb{R}, \mathbb{R})$ be such that

$$
f(u)=f_{\infty} u+\xi(u) .
$$

Clearly

$$
\lim _{u \rightarrow \infty} \frac{\xi(u)}{u}=0
$$

Let us consider

$$
L u-\lambda m(t) f_{\infty} u=\lambda m(t) \xi(u)
$$

as a bifurcation problem from infinity. We note that (80) is equivalent to (12) and (13).

Now, the results of Rabinowitz [25] for (80) can be stated as follows.

(i) For each integer $k \in\{1,2, \ldots, n\}$, there exists a continuum $\mathscr{D}_{k,+}^{v}$ of solutions to (80) meeting $\left(\lambda_{k,+} / r f_{\infty}, \infty\right)$.

(ii) For each integer $l \in\{1,2, \ldots, T-n\}$, there exists a continuum $\mathscr{D}_{l,-}^{\nu}$ of solutions to (80) meeting $\left(-\lambda_{l,-} / r f_{\infty}, \infty\right)$.

Lemma 21. Suppose that (H0), (H1), and (H2) hold. Then for $(\lambda, u) \in \mathscr{D}_{k,+}^{v} \cup \mathscr{D}_{l,-}^{v}$, we have

$$
\max _{t \in \mathbb{T}} u(t)>s_{2} .
$$

Proof. It is similar to the proof of Lemma 20, so we omit it. 
Lemma 22. Suppose (HO), (H1), and (H2) hold. Then

$$
\begin{gathered}
\left(\frac{\lambda_{k,+}}{f_{\infty}},+\infty\right) \subset \operatorname{Proj}_{\mathbb{R}}\left(\mathscr{D}_{k,+}^{\nu}\right), \quad \text { for } k=1,2, \ldots, n, \\
\left(-\infty, \frac{\lambda_{l,-}}{f_{\infty}}\right) \subset \operatorname{Proj}_{\mathbb{R}}\left(\mathscr{D}_{l,-}^{\nu}\right), \quad \text { for } l=1,2, \ldots, T-n .
\end{gathered}
$$

Proof. Firstly, we will prove $\left(\lambda_{k,+} / f_{\infty},+\infty\right) \subset \operatorname{Proj}_{\mathbb{R}}\left(\mathscr{D}_{k,+}^{\nu}\right)$. Take $\Lambda \subset \mathbb{R}$ as an interval such that $\Lambda \cap\left\{\left(\lambda_{j,+} / f_{\infty}\right) \mid j \in\right.$ $\{1,2, \ldots, n\}\}=\left\{\lambda_{k,+} / f_{\infty}\right\}$ and $\mathscr{M}$ is a neighborhood of $\left(\lambda_{k,+} / f_{\infty}, \infty\right)$ whose projection on $\mathbb{R}$ lies in $\Lambda$ and whose projection on $X$ is bounded away from 0 . Then by [25, Theorem 1.6 and Corollary 1.8], we have that either

(1) $\mathscr{D}_{k,+}^{v} \backslash \mathscr{M}$ is bounded in $\mathbb{R} \times E$ in which case $\mathscr{D}_{k,+}^{v} \backslash \mathscr{M}$ meets $\{(\lambda, 0) \mid \lambda \in \mathbb{R}\}$; or

(2) $\mathscr{D}_{k,+}^{v} \backslash \mathscr{M}$ is unbounded.

Moreover if (2) occurs and $\mathscr{D}_{k,+}^{v} \backslash \mathscr{M}$ has a bounded projection on $\mathbb{R}$, then $\mathscr{D}_{k,+}^{\nu} \backslash \mathscr{M}$ meets $(\widehat{\mu}, \infty)$ where $\widehat{\mu} \in$ $\left\{\lambda_{j,+} / f_{\infty}, j=1,2 \ldots, n\right.$ with $\left.j \neq k\right\}$.

Obviously Lemma 21 implies that (1) does not occur. So $\mathscr{D}_{k,+}^{v} \backslash \mathscr{M}$ is unbounded.

Lemma 19 guarantees that $\mathscr{D}_{k,+}^{v}$ is a component of solutions of (80) in $\Psi_{k}^{v}$ which meets $\left(\lambda_{k,+} / f_{\infty}, \infty\right)$. Therefore there is no $j \in\{1,2, \ldots, n\} \backslash\{k\}$ such that $\mathscr{D}_{k,+}^{\nu}$ also meets $\left(\lambda_{j,+} / f_{\infty}, \infty\right)$. Otherwise, there will exist $(\eta, y) \in \mathscr{D}_{k,+}^{\nu}$ such that $y$ has a multiple zero point $t_{0} \in(0, T+1)$; that is, $y\left(t_{0}\right)=0$ and $y\left(t_{0}-1\right) y\left(t_{0}+1\right) \geq 0$. However this contradicts with Lemma 19 , and consequently $\operatorname{Proj}_{\mathbb{R}}\left(\mathscr{D}_{k,+}^{\nu} \backslash \mathscr{M}\right)$ is unbounded. Thus

$$
\left(\frac{\lambda_{k,+}}{f_{\infty}},+\infty\right) \subset \operatorname{Proj}_{\mathbb{R}}\left(\mathscr{D}_{k,+}^{\nu}\right),
$$

and similarly we have

$$
\left(-\infty, \frac{\lambda_{l,-}}{f_{\infty}}\right) \subset \operatorname{Proj}_{\mathbb{R}}\left(\mathscr{D}_{l,-}^{\nu}\right) .
$$

Proof of Theorems 16, 17, and 18. From Lemmas 19-22 we have already completed the proof of Theorems 16 and 17 . We note that if $f_{\infty}=\infty$, then $\lambda_{k,+} / f_{\infty}=0$ and $\lambda_{l,-} / f_{\infty}=0$ which imply that the results of Theorem 18 hold.

\section{Conflict of Interests}

The authors declare that there is no conflict of interests regarding the publication of this paper.

\section{Acknowledgments}

This work is supported by NSFC (nos. 11061030, 11326127, and 11361054), SRFDP (no. 20126203110004), and Gansu Provincial National Science Foundation of China (no. 1208RJZA258).

\section{References}

[1] E. L. Ince, Ordinary Differential Equations, Dover, New York, NY, USA, 1926.

[2] A. Anane, O. Chakrone, and M. Moussa, "Spectrum of one dimensional $p$-Laplacian operator with indefinite weight," Electronic Journal of Qualitative Theory of Differential Equations, no. 17, pp. 1-11, 2002.

[3] P. Hess and T. Kato, "On some linear and nonlinear eigenvalue problems with indefinite weight function," Communications in Partial Differential Equations, vol. 5, pp. 999-1030, 1980.

[4] K. Bongsoo and K. Brown, "Existence of positive solutions for a class of indefinite weight semilinear elliptic boundary value problems," Nonlinear Analysis: Theory, Methods and Applications, vol. 39, no. 5, pp. 587-597, 2000.

[5] G. A. Afrouzi and K. J. Brown, "Positive mountain pass solutions for a semilinear elliptic equation with a sign-changing weight function," Nonlinear Analysis: Theory, Methods and Applications, vol. 64, no. 3, pp. 409-416, 2006.

[6] R. Ma and X. Han, "Existence and multiplicity of positive solutions of a nonlinear eigenvalue problem with indefinite weight function," Applied Mathematics and Computation, vol. 215, no. 3, pp. 1077-1083, 2009.

[7] R. Ma, J. Xu, and X. Han, "Global bifurcation of positive solutions of a second-order periodic boundary value problem with indefinite weight," Nonlinear Analysis: Theory, Methods and Applications, vol. 71, pp. 2119-2125, 2009.

[8] F. V. Atkinson, Discrete and Continuous Boundary Problems, Academic Press, New York, NY, USA, 1964.

[9] A. Jirari, Second-Order Sturm-Liouville Difference Equations and Orthogonal Polynomials, vol. 113, Memoirs of American Mathematical Society, 1995.

[10] R. P. Agarwal, M. Bohner, and P. J. Y. Wong, "Sturm-Liouville eigenvalue problems on time scales," Applied Mathematics and Computation, vol. 99, no. 2-3, pp. 153-166, 1999.

[11] Y. Shi and S. Chen, "Spectral theory of second-order vector difference equations," Journal of Mathematical Analysis and Applications, vol. 239, no. 2, pp. 195-212, 1999.

[12] M. Bohner, "Discrete linear Hamiltonian eigenvalue problems," Computers and Mathematics with Applications, vol. 36, no. 1012, pp. 179-192, 1998.

[13] G. Shi and R. Yan, "Spectral theory of left definite difference operators," Journal of Mathematical Analysis and Applications, vol. 337, no. 1, pp. 116-122, 2008.

[14] R. Ma, C. Gao, X. Han, and X. Chen, "Global structure of positive solutions of a discrete problem with sign-changing weight," Discrete Dynamics in Nature and Society, vol. 2011, Article ID 624157, 12 pages, 2011.

[15] R. P. Agarwal and J. Henderson, "Positive solutions and nonlinear eigenvalue problems for third-order difference equations," Computers and Mathematics with Applications, vol. 36, no. 1012, pp. 347-355, 1998.

[16] R. P. Agarwal and D. O’Regan, "Boundary value problems for discrete equations," Applied Mathematics Letters, vol. 10, no. 4, pp. 83-89, 1997.

[17] I. Rachůnková and C. C. Tisdell, "Existence of non-spurious solutions to discrete Dirichlet problems with lower and upper solutions," Nonlinear Analysis: Theory, Methods and Applications, vol. 67, no. 4, pp. 1236-1245, 2007.

[18] J. Rodriguez, "Nonlinear discrete Sturm-Liouville problems," Journal of Mathematical Analysis and Applications, vol. 308, no. 1, pp. 380-391, 2005. 
[19] S. S. Cheng and H.-T. Yen, "On a discrete nonlinear boundary value problem," Linear Algebra and Its Applications, vol. 313, no. 1-3, pp. 193-201, 2000.

[20] G. Zhang and W. Feng, "On the number of positive solutions of a nonlinear algebraic system," Linear Algebra and Its Applications, vol. 422, no. 2-3, pp. 404-421, 2007.

[21] R. Ma and H. Ma, "Positive solutions for nonlinear discrete periodic boundary value problems," Computers and Mathematics with Applications, vol. 59, no. 1, pp. 136-141, 2010.

[22] R. Ma and C. Gao, "Bifurcation of positive solutions of a nonlinear discrete fourth-order boundary value problem," Zeitschrift für Angewandte Mathematik und Physik, vol. 64, pp. 493-506, 2013.

[23] P. H. Rabinowitz, "Some global results for nonlinear eigenvalue problems," Journal of Functional Analysis, vol. 7, no. 3, pp. 487513, 1971.

[24] W. G. Kelley and A. C. Peterson, Difference Equations: An Introduction with Applications, Academic Press, New York, NY, USA, 1991.

[25] P. H. Rabinowitz, "On bifurcation from infinity, Journal of Differential Equations, vol. 14, no. 3, pp. 462-475, 1973. 


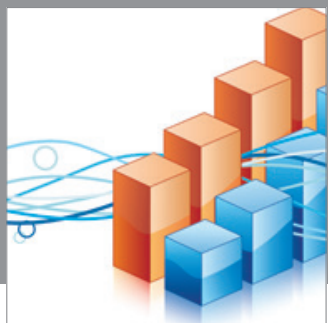

Advances in

Operations Research

mansans

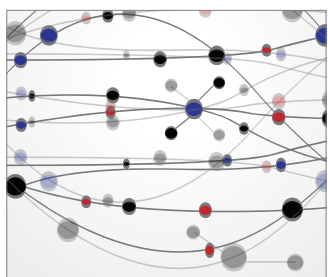

The Scientific World Journal
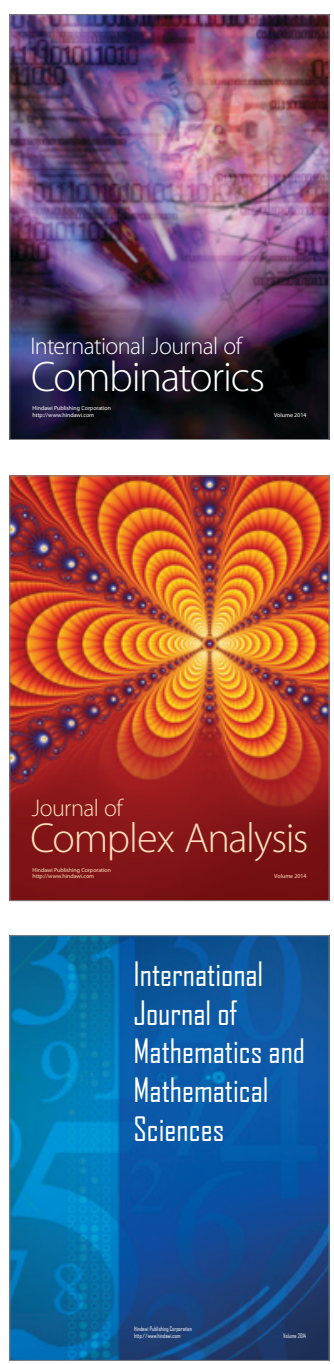
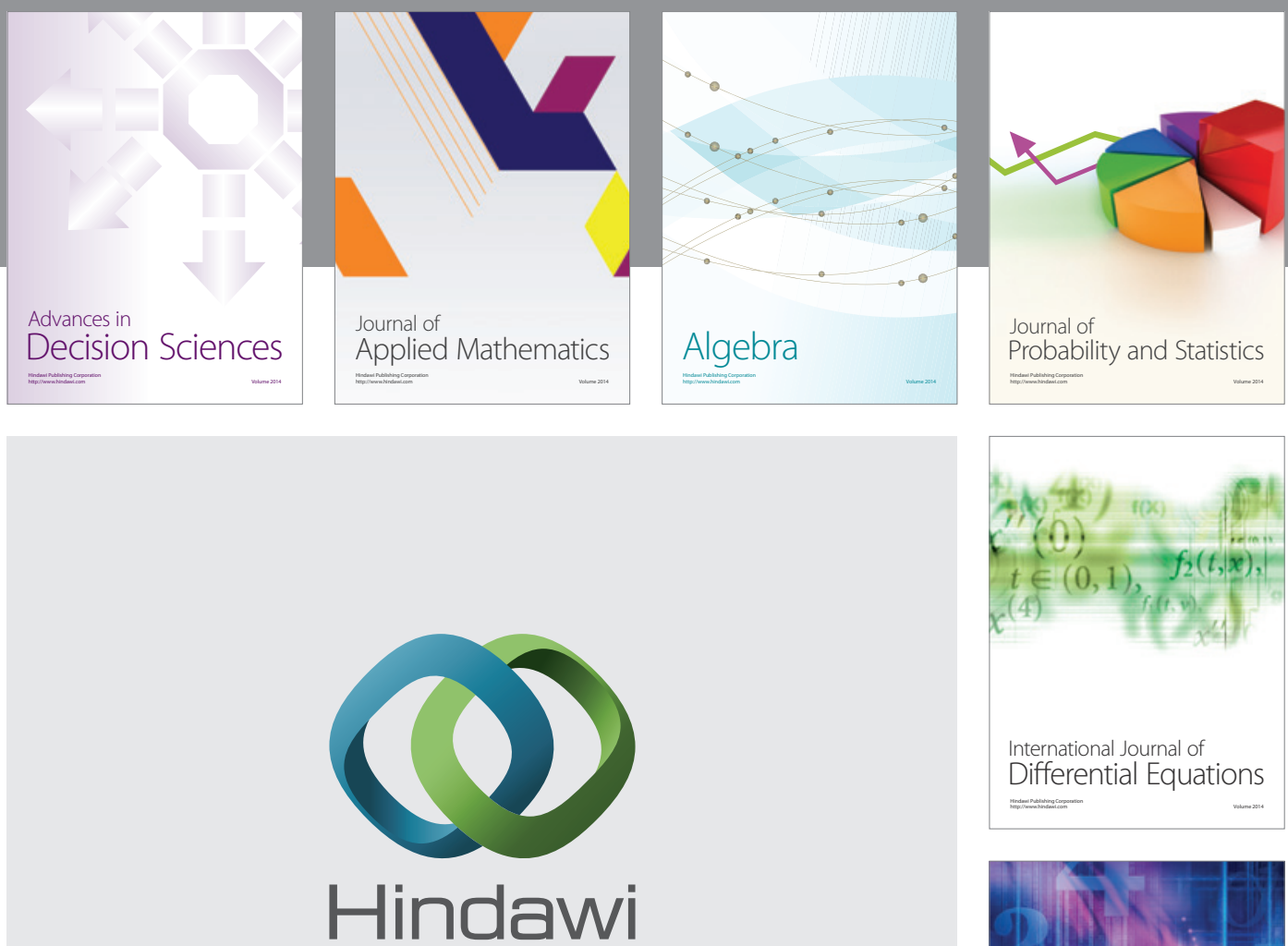

Submit your manuscripts at http://www.hindawi.com
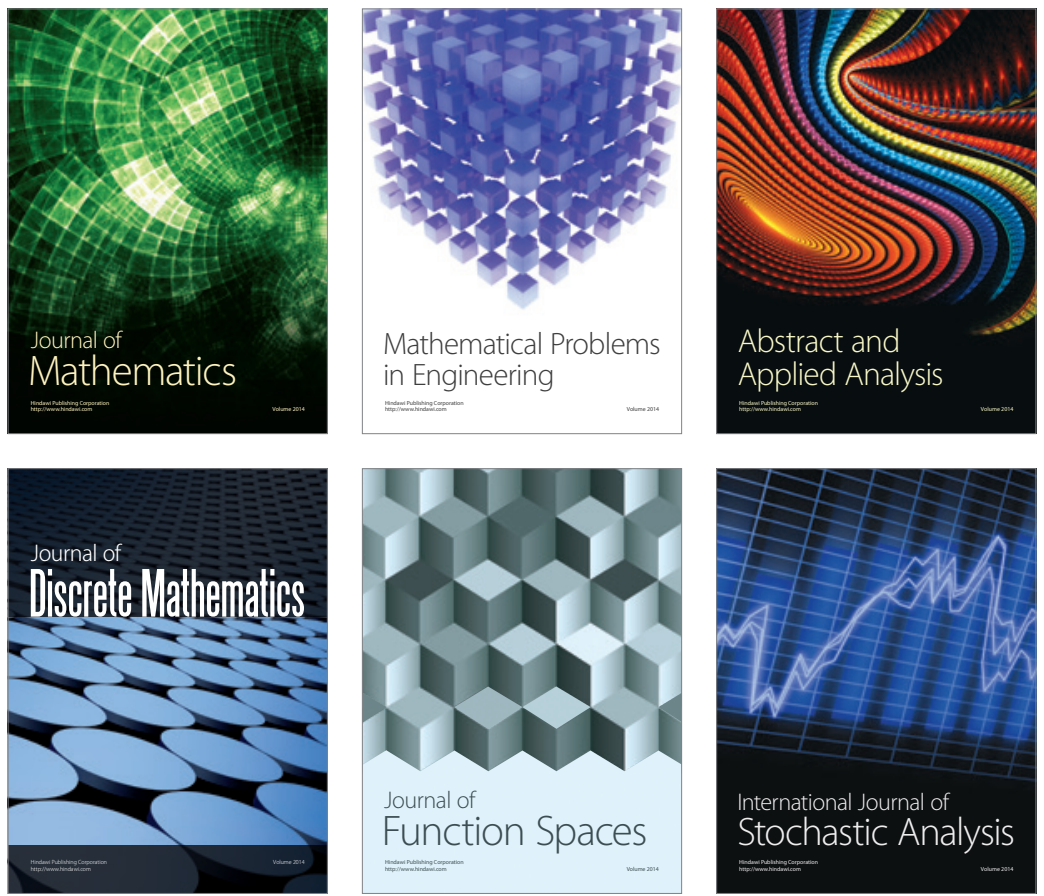

Journal of

Function Spaces

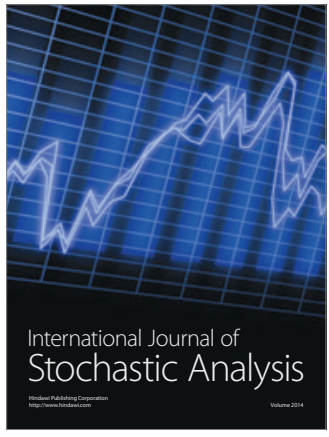

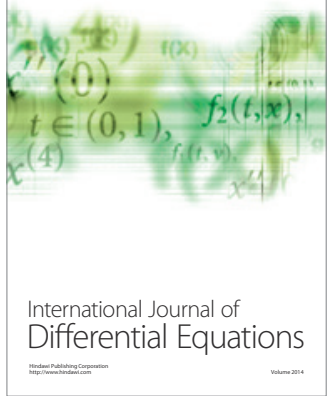
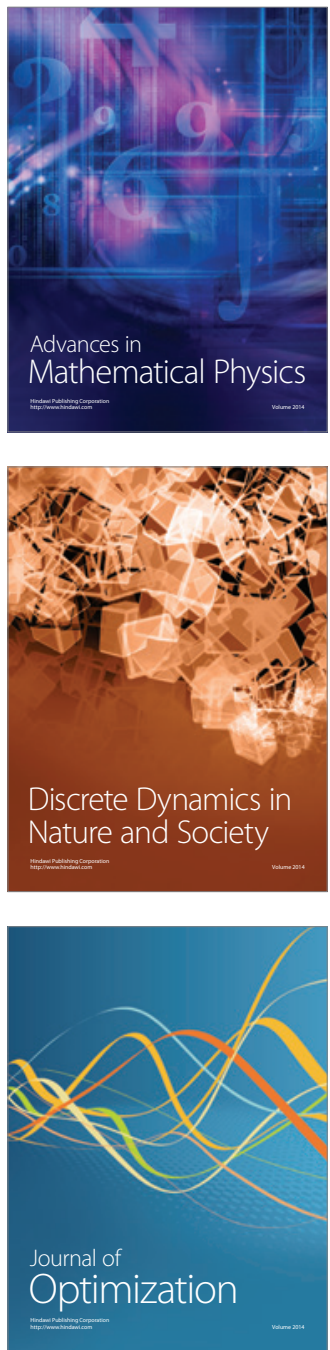